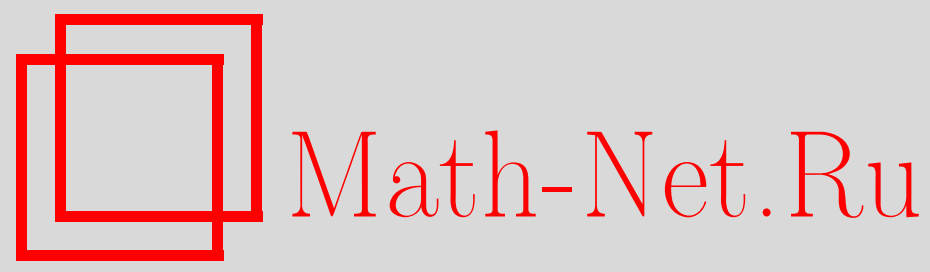

А. Г. Витушкин, Критерий представимости цепочки $\sigma$ процессов композицией треугольных цепочек, Матем. заметки, 1999, том 65, выпуск 5, 643-653

DOI: https://doi.org/10.4213/mzm1095

Использование Общероссийского математического портала Math-Net.Ru подразумевает, что вы прочитали и согласны с пользовательским соглашением http://www . mathnet.ru/rus/agreement

Параметры загрузки:

IP: 52.90 .164 .192

26 апреля 2023 г., 10:08:47 


\title{
КРИТЕРИЙ ПРЕДСТАВИМОСТИ ЦЕПОЧКИ $\sigma$-ПРОЦЕССОВ КОМПОЗИЦИЕЙ ТРЕУГОЛЬНЫХ ЦЕПОЧЕК
}

\section{А. Г. Витушкин}

\begin{abstract}
Для двумерного комплексного многообразия, содержащего цепочку сфер, получено условие на его топологические характеристики необходимое и достаточное для того, чтобы данная цепочка сфер была композицией треугольных цепочек. Получены формулы для вычисления этих характеристик. Рассматриваются некоторые следствия данного критерия, представляющие интерес в связи с гипотезой о якобиане.

Библиография: 3 названия.
\end{abstract}

Введение. Известно, что для всякого полиномиального отображения пространства $\mathbf{C}^{2}$ в себя, переходя к $\mathbf{C} P^{2}$ и выполняя конечное число $\sigma$-процессов (раздутий точек неопределенности отображения), можно построить компактное комплексное многообразие $M$, состоящее из $\mathbf{C}^{2}$ и конечного набора сфер, и такое, что данное отображение продолжается до голоморфного отображения $F$ всего этого пространства в $\mathbf{C} P^{1} \times \mathbf{C} P^{1}$. При этом указанньй набор сфер имеет структуру дерева, корнем которого является бесконечно удаленная сфера из $\mathbf{C} P^{2}$.

Всякую сферу дерева можно соединить с корневой некоторой цепочкой сфер. Эту цепочку можно выбрать таким образом, что после стягивания в точку каждой из сфер данного дерева, не попавших в эту цепочку, получится многообразие без особенностей, состоящее из $\mathbf{C}^{2}$ и этой цепочки сфер. Если, кроме того, число сфер в цепочке минимально, то в силу ацикличности дерева такая соединяющая цепочка единственна. (Подробнее об этом см. ниже в разделе 1.)

Предположим, что гипотеза о якобиане неверна, т.е. существует необратимое полиномиальное отображение $\mathbf{C}^{2}$ в себя с постоянньм не равным нулю якобианом. Разрешая на бесконечности особенности этого отображения, мы получим в данном случае разветвленную накрьвающую $(M, F)$ над $\mathbf{C} P^{1} \times \mathbf{C} P^{1}$. Пусть $M^{*}=F^{-1}\left(\mathbf{C}^{2}\right)$ - часть поверхности $M$, лежащая над $\mathbf{C}^{2}$. Поверхность $M^{*}$ состоит из многообразия, изоморфного $\mathbf{C}^{2}$ и конечного набора кривых, изоморфных $\mathbf{C}^{1}$ или сфере.

Рассмотрим простейший возможньй случай, когда $M^{*}$ состоит всего лишь из двух клеток, первая из которых изоморфна $\mathbf{C}^{2}$, а вторая $-\mathbf{C}^{1}$. На первой клетке отображение локально обратимо. Вторая клетка является кривой ветвления.

Ясно, что группа одномерных гомологий с целочисленньми коэффициентами поверхности $M^{*}$ тривиальна, а групша двумерных гомологий - это свободная групша ранга 1.

Работа выполнена при финансовой поддержке Российского фонда фундаментальных исследований. 
Образующую этой группы можно построить, например, так. Фиксируем малый диск, трансверсально пересекающий кривую ветвления. Границу этого диска продеформируем в точку, оставаясь все время внутри первой клетки. Получилась сфера. Ориентацию на ней выберем так, чтобы ее индекс пересечения с кривой ветвления был равен -1 . Обозначим эту сферу через $S$ и будем называть ее пробной сферой или тест-сферой. Название оправдьвается тем, что свойства этой сферы во многом определяют свойства накрывающей поверхности. Отметим, что в качестве пробной поверхности можно выбирать не обязательно эту сферу, а любую поверхность, которая является образующей группы двумерных гомологий. Такие поверхности при подходящем выборе их ориентации гомологичны друг другу, и, как будет видно из дальнейшего, интересующие нас топологические свойства таких поверхностей одинаковы.

Далее, с одной стороны, зная, что поверхность $M^{*}$ накрьвает относительно просто устроенное пространство, а именно $\mathbf{C}^{2}$, и то, что кривая ветвления изоморфна $\mathbf{C}^{1}$, мы можем в некоторых случаях вычислить топологические характеристики пробной сферы. С другой стороны, помня, что речь идет о необратимом полиномиальном отображении с невырожденным якобианом, мы можем указать в дереве сфер ту сферу, которая вся, кроме одной ее точки, переводится отображением $F$ в $\mathbf{C}^{2}$ и является при этом кривой ветвления. Рассмотрим минимальную цепочку $\sigma$, соединяющую корневую сферу дерева с этой сферой. Как будет видно из дальнейшего (см. разделы 2, 3), характеристики пробной сферы, которые нам удалось вычислить, дают некоторую информацию о структуре этой соединяющей цепочки. Это обстоятельство ограничивает класс многочленов, который может дать контрпример к гипотезе о якобиане.

Рассмотрим конкретный пример. В [1] и [2] построены примеры экзотических разветвленных накрываюших, которые по своим топологическим свойствам подходят для того, чтобы быть контрпримером к гипотезе о якобиане. Разумеется, какой-либо из этих примеров или их аналог может стать контрпримером лишь при условии, что его удастся реализовать посредством полиномиального отображения. Априори, искомоеполиномиальное отображение может быть устроено сколь угодно сложно. Вычисление характеристик пробной сферы этих накрывающих позволило упростить задачу. А именно, в [3] доказано, что для этих накрьвающих индекс самопересечения пробной сферы равен -3 , а значение первого класса Черна на этой сфере равно 1. С другой стороны, из утверждения 1 (см. ниже раздел 2) следует, что при таких значениях характеристик пробной сферы искомоеполиномиальное отображение должно быть устроено достаточно просто. А именно, при разрешении особенностей отображения получается,что цепочка, соединяюшая корневую сферу дерева с кривой ветвления, состоит всего лишь из 4-х $\sigma$-процессов.

Изучение пробных поверхностей, вывод формул для вычисления их характеристик и некоторые применения этих формул составляют предмет данной статьи.

Автор выражает искреннюю благодарность Н. Кружилину, С. Немировскому и С. Оревкову за ценные советы при обсуждении статьи.

1. Необходимые определения. Цепочку сфер принято назьвать минимальной иепочкой (минимальной цепочкой $\sigma$-процессов), если эта цепочка получена следующим образом. Пусть $\widetilde{M}_{0}$ - двумерное комплексное многообразие и $\widetilde{\sigma}_{0} \subset \widetilde{M}_{0}-$ сфера такая, что индекс самопересечения $\widetilde{\sigma}_{0}^{2}= \pm 1$. На этой сфере выберем какую-либо точку и раздуем эту точку несколько раз. На последней из полученных таким образом сфер опять выберем какую-либо точку и раздуем ее несколько раз ... Повторим эту процедуру конечное число раз. Мы получим цепочку комплексных многообразий $M_{0}, \ldots, M_{l}$ и це- 
почку сфер $\widetilde{\sigma}_{0}, \ldots, \widetilde{\sigma}_{l}$. При всяком $i \widetilde{\sigma}_{i} \subset M_{i}$. Образы этих сфер в пространстве $M=M_{l}$ обозначим через $\sigma_{0}, \ldots, \sigma_{l}$. Это и есть искомая цепочка сфер. Сферу $\widetilde{\sigma}_{0}$ мы будем называть корневой сферой данной цепочки и будем всюду далее предполагать, что эта сфера комплексно-аналитична (это условие позволит нам, в частности, говорить о естественной ориентации данной сферы). Многообразия и сферы пронумерованы в порядке их появления. Отметим, что при всяком $i \neq 0, l{\sigma_{i}}^{2} \neq-1$, и потому никакую из этих сфер нельзя стянуть в точку так, чтобы объемлющее пространство оставалось без особенностей. Именно это свойство цепочки оправдьвает ее название - минимальная. Условимся, что в дальнейшем, говоря о цепочке сфер, мы будем всегда предполагать, что эта иепочка минимальна, напоминая о минимальности лишь в основных формулировках.

Цепочку принято назьвать линейной, если каждая сфера из этой цепочки пересекается не более, чем с двумя другими ее сферами. Ясно, что такую цепочку сфер можно перенумеровать так, чтобы всякая сфера, кроме первой и последней, пересекалась с двумя сферами - с предыдущей и последующей. При этом сфера, имевшая нулевой индекс в исторической нумерации (нумерации в порядке появления сфер), будет иметь и в новой, геометрической нумерации также нулевой индекс. Но последняя сфера исходной цепочки после перенумерации может оказаться где-то внутри цепочки. Эту сферу мы отметим звездочкой и обозначим через $\gamma_{\kappa}^{*}$. Получилась цепочка $\gamma_{0}, \ldots, \gamma_{k}^{*}, \ldots, \gamma_{l}$.

Если $k=l$, то такую цепочку мы будем называть әлементарной цепочкой, а если $k<l$-составной иепочкой . (Составная цепочка состоит из двух цепочек - от 0 до $k$ и от $k$ до l.) Число $k$ будем называть порядком чепочки, а число $l$ - ее длиной .

Нетрудно проверить, что всякая составная цепочка получается путем выполнения следующей последовательности $\sigma$-процессов. На сфере $\widetilde{\gamma}_{0}$ выбирается какая-либо точка $p_{1}$ и она раздувается $r_{1} \geqslant 2$ раз. На всяком следуюшем шаге раздуваемая точка выбирается однозначно. А именно, на $i$-м шаге $r_{i} \geqslant 1$ раз раздувается точка $p_{i} \neq p_{i-1}$, являющаяся пересечением 2-х сфер.Одна из них - это последняя сфера, полученная на $(i-1)$-м шаге. Вторая - в зависимости от порядкового номера раздутия, это или предпоследняя сфера, полученная на $(i-1)$-м шаге (если точка раздувается в первьй раз), или сфера, полученная предшествующим раздутием на $i$-м шаге. Общее число шагов равно порядку цепочки, т. е. числу $k$. Построение элементарной цепочки отличается тем, что на каждом шаге раздувается точка, принадлежащая только последней из полученных сфер, и раздувается она только один раз.

Отметим, что однозначность выбора раздуваемой точки при построении составной цепочки определяется условием минимальности цепочки. Если раздувать непоследнюю из полученных сфер, то после вьполнения всех раздутий внутри цепочки найдется сфера с индексом самопересечения -1 , что противоречит условию минимальности.

Цепочку будем назьвать составной чепочкой простейшего типа порядка $k$, если $r_{1}=2$ и при $i=2, \ldots, k r_{i}=1$, или, что то же самое, если длина этой цепочки $l=k+1$.

Мы будем разбивать сложные цепочки сфер на части и будем называть эти части

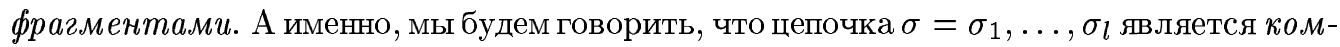
позичией фрагментов $\phi_{1}, \ldots, \phi_{n}$, если при всяком $i \phi_{i}$ - это линейная цепочка; при $i<n$ последняя сфера цепочки $\phi_{i}$ является корневой для цепочки $\phi_{i+1}$; сфера $\sigma_{0}$ является первой сферой фрагмента $\phi_{1}$, a $\sigma_{l}$ - последней сферой фрагмента $\phi_{n}$. Разбиение на фрагменты будет единственным, если потребовать, чтобы всякая сфера цепочки $\sigma$, пересекающаяся с тремя другими ее сферами, была последней сферой одного из фрагментов и одновременно первой сферой следующего за ним фрагмента. Разбиение на фрагменты 
позволяет свести вычисление топологических характеристик пространства, содержащего данную цепочку, к описанию свойств фрагментов этой цепочки.

Цепочка назьвается треугольной иепочкой, если она является композицией двух линейных цепочек, таких, что первая из них - это составная цепочка простейшего типа некоторого порядка $k \geqslant 1$, а вторая - элементарная цепочка длины $k$. Выбор названия оправдывается наличием аналогичного термина, относяшегося к полиномиальным преобразованиям. Преобразование назьвается треугольным, если оно имеет вид: $x \rightarrow x+f(y), y \rightarrow y$, где $f$ - многочлен, зависяший только от $y$. Такое преобразование геометрически реализуется переходом от $\mathbf{C}^{2}$ к $\mathbf{C} P^{2}$ с последующим вьполнением треугольной цепочки $\sigma$-процессов и стягиванием сфер этой цепочки, начиная с первой из них.

2. Формулировка результата. Пусть $\sigma=\sigma_{0}, \sigma_{1}, \ldots, \sigma_{l}$ - минимальная цепочка, лежащая в многообразии $M$. Положим $M^{*}=M \backslash \bigcup_{i=0}^{l-1} \sigma_{i}$ и $\sigma^{*}=\sigma_{l} \backslash \bigcup_{i=0}^{l-1} \sigma_{i}$. Фиксируем гладко погруженную в $M^{*}$ ориентированную поверхность $S$ вещественной размерности 2 и такую, что индекс пересечения $S \cdot \sigma^{*}=-1$. Нашей целью является изучение связи структуры цепочки $\sigma$ с топологическими характеристиками поверхности $S$. Имеется в виду индекс самопересечения $S^{2}$ и значение $H$ первого класса Черна на этой поверхности.

Если $M^{*} \backslash \sigma^{*}=\mathbf{C}^{2}$, то $S^{2}$ и $H$ не зависят от выбора $S$. Для произвольного многообразия $M^{*}$ это, вообще говоря, неверно, и потому далее относительно поверхности $S$ будем предполагать, что она лежит в достаточно малой окрестности цепочки $\sigma$. При этом условии указанные топологические характеристики не будут зависеть от выбоpa $S$. Всякую такую поверхность $S$ мы будем называть пробной поверхностью данной цепочки.

Поверхность $S$ с вьшеперечисленными свойствами существует и может быть получена в виде объединения $S=\bigcup_{i=0}^{l} x_{i} \sigma_{i}$. Вектор $x=\left(x_{0}, \ldots, x_{l}\right)$ находится из уравнения $C x=y$, где $C=\left(-\sigma_{i} \cdot \sigma_{j}\right)$ - взятая со знаком “-" матрица пересечений сфер из цепочки $\sigma$ и $y=(0, \ldots, 0,1)$. В рассматриваемой ситуации определитель $d=\operatorname{det}(C)=-\widetilde{\sigma}_{0}^{2}= \pm 1$ не равен 0 и потому решение существует.

ТЕоремА. Пусть $\sigma=\sigma_{0}, \sigma_{1}, \ldots, \sigma_{l}-$ минимальная цепочка сфер, $\widetilde{\sigma}_{0}-$ корневая сфера иепочки, $S$ - пробная поверхность этой иепочки и $H$ - значение первого класса Черна на S. Тогда верно следующее:

1) если $S^{2}=1$ и $H=-3$, то иепочка $\sigma$ является композицией треугольных иепочек и при этом $\widetilde{\sigma}_{0}^{2}=1$ (и, следовательно, $\left.\sigma_{0}^{2}=-1\right)$;

2) если цепочка б является композицией треугольных иепочек и $\widetilde{\sigma}_{0}^{2}=1$, то $S^{2}=1$ u $H=-3$.

Эта теорема появилась в процессе доказательства следующего утверждения.

УТВЕРЖДЕНИЕ 1. Если иепочка $\sigma$ минимальна и для нее $H+S^{2}=-2 u|H| \leqslant 3$, то такая иепочка является композичией нескольких треугольных иепочек и одной (завершающей) әлементарной иепочки длины $H+3$, и при этом получается, что $\tilde{\sigma}_{0}^{2}=1$.

Отметим, что при некоторых значениях $H>3$ утверждение 1 неверно. Простейший контрпример - это цепочка, имеющая корнем бесконечно удаленную сферу в $\mathbf{C} P^{2}$, являющаяся композицией 4 -х фрагментов $\phi_{1}, \phi_{2}, \phi_{3}, \phi_{4}$, где $\phi_{1}, \phi_{2}$ и $\phi_{4}$ - это составные 
цепочки простейшего типа порядка 1 , а $\phi_{3}$ - это элементарная цепочка длины 7 . Из лемм 3 и 6 (см. ниже) легко получить, что $H=4$ и $S^{2}=-6$.

3. Вычисление топологических характеристик пробной поверхности. Наряду с изложенной в предыдущем разделе известной формой записи поверхностей мы рассмотрим здесь еще одну форму записи, построив для этого специальньй ортогональный базис сфер. При рассмотрении геометрических свойств поверхностей эта форма в ряде случаев оказывается более удобной, чем первая. Эти два способа записи пробной поверхности вместе дают возможность, как будет видно из дальнейшего, свести вычисление топологических характеристик цепочки сфер к вычислению аналогичных характеристик фрагментов цепочки.

Сопоставим заданной цепочке сфер $\sigma=\sigma_{0}, \ldots, \sigma_{l}$ цепочку сфер $\omega=\omega_{0}, \ldots, \omega_{l}$. Для этого на всяком шаге построения первой цепочки, получив сферу $\widetilde{\sigma}_{i}$, прежде, чем вьполнять следующий шаг конструкции, переведем посредством малой гладкой деформации сферу $\widetilde{\sigma}_{i}$ в какую-либо сферу $\widetilde{\omega}_{i}$ и примем в качестве $\omega_{i}$ образ сферы $\widetilde{\omega}_{i}$ в многообразии $M_{l}$. При $i \neq 0$ ориентация сферы $\widetilde{\omega}_{i}$ и, соответственно, $\omega_{i}$, задается поднятием ориентации со сферы $\widetilde{\sigma}_{i}$. Если $\widetilde{\sigma}_{0}^{2}=-1$, то ориентация сферы $\widetilde{\omega}_{0}$ определяется точно так же, а в случае, если $\widetilde{\sigma}_{0}^{2}=1$, ориентация сферы $\widetilde{\omega}_{0}$, а соответственно, и сферы $\omega_{0}$, выбирается противоположной ориентации сферы $\widetilde{\sigma}_{0}$.

Лемма 1. Для всякой минимальной цепочки б соответствующая цепочка $\omega$ можст быть выбрана таким образом, чтобь выполнялось следующее:

1) сферы $\left\{\omega_{j}\right\}$ попарно не пересекаются;

2) $п р и j=i \quad \omega_{j} \cdot \sigma_{i}=-1$;

3) если при построении цепочки $\sigma$ точка сферы $\widetilde{\sigma}_{i}$ раздувалась $m$ раз, то при $j=i+1, \ldots, i+m \omega_{j} \cdot \sigma_{i}=1, u$ при всяком другом значении $j \omega_{j} \cdot \sigma_{i}=0 ;$

4) при всяких $i, j$ пересечение сфер $\sigma_{i} u \omega_{j}$ или пусто или состоит из одной точки.

Доказательство леммы опускаем, отметим лишь одну деталь, касающуюся п. 3, которая при беглом чтении может оказаться незамеченной. Если, например, точку сферы $\tilde{\sigma}_{0}$ мы раздували 3 раза, то каждая из сфер $\omega_{j}(j=1,2,3)$ пересекает сферу $\sigma_{0}$, но при этом сферы $\sigma_{2}$ и $\sigma_{3}$ имеют еще две точки пересечения, а именно, $\omega_{2}$ пересекает $\sigma_{1}$ и $\omega_{3}$ пересекает сферу $\sigma_{2}$, поскольку на $\widetilde{\sigma}_{1}$ и $\widetilde{\sigma}_{2}$ имеется по одной точке, которые раздувались.

ЛЕмма 2. Для всякой минимальной иепочки $\sigma$ существует, и притом единственный, набор натуральных чисел $n_{i}(i=0,1, \ldots, l)$ такой, что $n_{l}=1$ и для поверхности $S=\bigcup_{i=1}^{l} n_{i} \cdot \omega_{i}$ выполняются равенства $S \cdot \omega_{l}=-1$ и при всяком $i<l S \cdot \omega_{i}=0$. При этом $n_{i}=\sum_{j} n_{j}$. Суммирование производится по всем значениям индекса $j$ таким, что $\sigma_{i} \cdot \omega_{j}=1$ (см. лемму 1$)$.

Доказательство опускаем. Указанная в лемме 2 поверхность $S$ с помощью стандартной процедуры (соединением элементов связной суммы трубочками) может быть превращена в гладкую поверхность. В данном случае эта поверхность является гладко погруженной сферой, пересекающей цепочку $\sigma$ лиш в одной точке, а именно, в точке из $\sigma_{l}$.

УТВЕРЖДЕНИЕ 2. Пусть $\sigma=\sigma_{0}, \ldots, \sigma_{l}$ - это минимальная иепочка, $d= \pm 1$ значение определителя пересечений матрицы $C$ этой иепочки (см. раздел 2) $u$ 
$\left\{n_{i}\right\}$ - коэффициенты разложсения пробной поверхности (см. лемму 2). Тогда топологические характеристики поверхности $S$ можно вычислять по формулам:

1) индекс самопересечения $S^{2}=-d \cdot n_{0}^{2}-\sum_{i=1}^{l} n_{i}^{2}$;

2) индекс самопересечения в нормальном расслоении $e=-d \cdot n_{0}-\sum_{i=1}^{l} n_{i}$;

3) значение на $S$ первого класса Черна $H=(1-2 d) n_{0}+\sum_{i=1}^{l} n_{i}$.

ДокАЗАТЕльство. Зададим $S$ формулой, указанной в лемме 2 . Сфера $\omega_{i}$ включена в $S$ в $n_{i}$ экземплярах. Экземпляры этих сфер, соответствующие различным значениям индекса $i$, попарно не пересекаются (см. лемму 1 ), и поэтому индекс самопересечения, а также индекс самопересечения в нормальном расслоении, набирается лишь за счет пересечения экземпляров с одним и тем же значением индекса. Подсчитывая эти пересечения с учетом того, что $\widetilde{\omega}_{0}^{2}=-d$ и при $i>0 \widetilde{\omega}_{i}^{2}=-1$, легко получить первые два равенства из утверждения 2.

Напомним, что если индекс самопересечения сферы, имеющей комплексную структуру, равен $r$, то при естественном выборе ориентации сферы значение первого класса Черна равно $r+2$. Каждая из сфер $\omega_{i}$ получена малой гладкой деформацией сферы с комплексной структурой, и поэтому, учитывая правило, с помощью которого задавалась ориентация на сферах $\omega_{i}$, получаем при $i>0 H\left(\omega_{i}\right)=1$ и $H\left(\omega_{0}\right)=-(1-2 d)$. Тем самым, вспомнив, что сфера $\omega_{i}$ входит в $S$ с коэффициентом $n_{i}$, получаем третье равенство утверждения 2. Утверждение доказано.

4. Формулы для линейной цепочки. Некоторые формулы, которые будут приведены ниже в леммах 3-7, известны и в том или ином виде могут быть найдены в литературе. Исключение составляет, быть может, лишь лемма 5. Доказательство этих формул достаточно просто. Поэтому мы приводим доказательства, вместо того,чтобы искать ссылки и заниматься сравнением формулировок.

Пусть $\sigma=\sigma_{0}, \ldots, \sigma_{l}$ - минимальная линейная цепочка, пронумерованная в историческом порядке (см. раздел 1$),$ и $S=\bigcup_{i=0}^{l} x_{i} \sigma_{i}$ - пробная поверхность этой цепочки. Определим для иепочки два параметра $p$ и $q$. Чтобы сделать это, запишем цепочку $\sigma$ в геометрическом порядке $\gamma=\gamma_{0}, \ldots, \gamma_{k}^{*}, \ldots, \gamma_{l}$. Поверхность $S$ после этого перепишется в виде $S=\bigcup_{i=0}^{l} y_{i} \gamma_{i}$. Ясно, что $\gamma_{0}=\sigma_{0}$ и $\gamma_{k}^{*}=\sigma_{l}$. Следовательно, $y_{0}=x_{0}$ и $y_{k}=x_{l}$. Пусть $C^{*}=\left(-\gamma_{i} \cdot \gamma_{j}\right)$ - матрица пересечений цепочки $\gamma_{0}, \ldots, \gamma_{k-1}$ и $C^{* *}=\left(-\gamma_{i} \cdot \gamma_{j}\right)-$ матрица пересечений цепочки $\gamma_{k+1}, \ldots, \gamma_{l}$. Обозначим через $p$ определитель матрищы $C^{* *}$ и через $q$ определитель матрицы $C^{*}$. В тех случаях, когда какая-либо из матриц пуста, т.е. ее размер равен 0 , будем считать, что определитель такой матрицы равен 1 . Через $d$, как и ранее (см. раздел 2$)$, будем обозначать определитель $d=\operatorname{det}(C)=-\widetilde{\gamma}_{0}^{2}= \pm 1$.

ЛЕмма 3. Для цепочек $\sigma$ и $\gamma$ и их пробной поверхности $S$ выполняется следуюuяee:

1) значение на $S$ первого класса Черна $H=d(p+q-1)$;

2) индекс самопересечения $S^{2}=-d p q$;

3) $x_{0}=y_{0}=d p$ и $y_{l}=d q u x_{l}=y_{k}=d p q$, при этом если $\sigma$ является составной иепочкой, то $p \geqslant 2$.

ДоказАтЕльство. Вектор $Y=\left(y_{0}, \ldots, y_{l}\right)$ является решением уравнения $C \cdot Y=$ $Y^{*}$, где $C-(-)$ матрица пересечений цепочки $\gamma$, а $Y^{*}$ - это вектор, $k$-я координата которого равна 1 , а все остальные равны нулю. При такой форме записи поверхности ее 
скалярньй квадрат вычисляется по формуле $S^{2}=Y^{t}(-C) Y=-Y^{t} Y^{*}=-y_{k}$. A поскольку дополнительный минор элемента матрицы $C$, которьй стоит на пересечении $k$-й строки и $k$-го столбца, равен $\left|C^{* *}\right| \cdot\left|C^{*}\right|=p q$, то по формуле Крамера получаем $S^{2}=-p q / d=-d p q$.

Далее, так как при всяком $i H\left(\gamma_{i}\right)=\gamma_{i}^{2}+2$, то

$$
\begin{aligned}
H & =\sum_{i=0}^{l} y_{i} H\left(\gamma_{i}\right)=\sum_{i=0}^{l} y_{i}\left(1+\gamma_{i}^{2}+1\right) \\
& =y_{0}+\left(y_{0} \gamma_{0}^{2}+y_{1}\right)+\sum_{i=1}^{l-1}\left(y_{i-1}+y_{i} \gamma_{i}^{2}+y_{i+1}\right)+\left(y_{l-1}+y_{l} \gamma_{l}^{2}+y_{l}\right) \\
& =y_{0}+S \gamma_{0}+\sum_{i=1}^{l-1} S \gamma_{i}+S \gamma_{l}+y_{l}=y_{0}+S \gamma_{k}^{*}+y_{l}=y_{0}-1+y_{l} .
\end{aligned}
$$

Подставляя в матрицу $C$ вместо первого столбца вектор $Y^{*}$, а затем вместо последнего столбца опять же вектор $Y^{*}$ и вычисляя определители получившихся матриц, мы получим из формулы Крамера, что $y_{0}=d p$, а $y_{l}=d q$. И это дает нам нужное равенство для $H$. Проверку пункта 3 опускаем. Лемма доказана.

Пусть задан набор натуральных чисел $r_{1}, \ldots, r_{k}$, определяющий составную цепочку $\gamma=\gamma_{0}, \ldots, \gamma_{k}^{*}, \ldots, \gamma_{l}$. Зафиксируем $i$ и рассмотрим цепочку, которая получается в процессе построения цепочки $\gamma$ после вьполнения $i$-го шага.Эта цепочка определяется набором чисел $r_{1}, \ldots, r_{i}$. Соответствующие ей параметры $p$ и $q$ обозначим через $p_{i}, q_{i}$ $(i=1, \ldots, k)$. Пополним этот список параметров парой $p_{0}=1$ и $q_{0}=1$.

ЛЕмма 4. Для всякой составной иепочки, определяемой набором чисел $r_{1}, \ldots, r_{k}$, выполняются следующие рекурентные соотношения:

$p_{1}=r_{1} p_{0} u q_{1}=r_{1} q_{0}+d ; p_{i}=r_{i} p_{i-1}+\left(r_{i-1}-1\right) p_{i-2}+\cdots+\left(r_{1}-1\right) p_{0} \quad(i=2,3 \ldots, k) ;$ $q_{i}=r_{i} q_{i-1}+\left(r_{i-1}-1\right) q_{i-2}+\cdots+\left(r_{1}-1\right) q_{0}+d \quad(i=2,3, \ldots, k)$.

Доказательство леммы опускаем, поскольку оно легко сводится к последовательному вычислению определителей, задающих параметры $p_{i}$ и $q_{i}$.

ЛЕмма 5. Для всякой составной иепочки $\gamma$ порядка $k$ ее параметры $р$ и q удовлетворяют неравенствам:

1) если $d=1$, mo $p+k \leqslant q \leqslant 2 p-1$;

2) если $d=-1$, mo $1 \leqslant q \leqslant 2 p-2 k-1$.

Кроме того, при любом значении $d$, если хотя бы одно из двух неравенств, соответствующих данному значению $d$, обращается в равенство, то $\gamma$ является иепочкой простейшего типа, и обратно, если $\gamma$ является иепочкой простейшего типа, то оба неравенства, соответствующие данному значению $d$, обращаются в равенства.

Лемма доказывается индукцией по $k$. При $k=1 p_{1}=r_{1}$ и $q_{1}=r_{1}+d$, и в этом случае утверждение леммы очевидно. Пусть теперь $k>1$. Воспользуемся рекурентными формулами из леммы $4: q_{k}=r_{k} q_{k-1}+\cdots+d$.При $d=1$ в силу предположения индукции при всех $i=1,2, \ldots, k-1 q_{i} \leqslant 2 p_{i}-1$. Следовательно, $q_{k} \leqslant r_{k}\left(2 p_{k-1}-1\right)+$ 
$r_{k-1}\left(2 p_{k-2}-1\right)+\cdots+d$. Используя первое равенство леммы 4 , выразим $p_{k-1}$ через $p_{k}$. Получаем $p_{k-1}=r_{k}^{-1}\left(p_{k}-\left(r_{k-1}-1\right) p_{k-2}-\cdots\right)$. Исключая с помощью этого равенства $p_{k-1}$ из правой части неравенства $q_{k} \leqslant r_{k}\left(2 p_{k-1}-1\right)+\cdots$, приводя подобные члены и убирая некоторые заведомо неположительные слагаемые, получаем $q_{k} \leqslant 2 p_{k}-1-\left(r_{1}-1\right) p_{0}+d$. А так как $d=1$, а $r_{1} \geqslant 2$, то получаем искомое неравенство $q \leqslant 2 p-1$.

Три оставшиеся неравенства доказываются аналогично. Доказательство второй части леммы проведем на примере того же неравенства. Так как $q_{i} \leqslant 2 p_{i}-1, q_{i}$ может быть записано в виде $q_{i}=2 p_{i}-1-\varepsilon_{i}$, где $\varepsilon_{i} \geqslant 0$. Повторяя приведенные вьше рассуждения, получим $q_{k}=2 p_{k}-r_{k}\left(1+\varepsilon_{k}\right)-\cdots-2\left(r_{i}-1\right)\left(1+\varepsilon_{i}\right)-\cdots-\left(r_{1}-1\right) p_{0}+d$. Если правая часть равна $2 p_{k}-1$, т.е. если неравенство $q \leqslant 2 p-1$ обратилось в равенство, то видно, что $r_{1}=2$ и при всех $i=2,3, \ldots, k r_{i}=1$, т.е. данная цепочка является цепочкой простейшего типа. С другой стороны, если известно, что составная цепочка $\gamma$ является цепочкой простейшего типа, то опять же индукцией по $k$ доказьвается, что неравенсво обращается в равенство. Лемма доказана.

5. Формулы для композиции цепочек. Пусть $\sigma=\sigma_{0}, \sigma_{1}, \ldots$ - это минимальная цепочка, являющаяся композищией трех фрагментов $\phi_{0}, \phi_{1}, \phi_{2}$. Здесь $\phi_{0}$ - это цепочка, последняя сфера которой имеет индекс самопересечения $-1, \phi_{1}$ - составная цепочка порядка $k \geqslant 1$ с параметрами $p$ и $q$ и $\phi_{2}-$ это элементарная цепочка длины $l \geqslant 0$. Пусть, далее, $S_{0}$ - это пробная поверхность фрагмента $\phi_{0}$ и $S$ - пробная поверхность всей цепочки $\sigma ; H_{0}$ и $H-$ значения первого класса Черна на $S_{0}$ и $S$, соответственно.

ЛемМа 6. Виполняются равенства:

1) $S^{2}=S_{0}^{2} p^{2}+p^{2}-p q-l$

2) $H=H_{0} p+q-1+l$.

ДокАЗАТЕльство. Пусть $S_{1}$ - пробная поверхность фрагмента $\phi_{1}$, заданная в виде $S_{1}=\bigcup_{i=1}^{n} y_{i} \gamma_{i}$ (см. лемму 3$)$. В качестве пробной поверхности фрагмента $\phi_{0}$ примем поверхность вида $S_{0}=\bigcup_{i=0}^{m} n_{i} \omega_{i}$ (см. лемму 2).

Рассмотрим сначала случай $l=0$. В этом случае цепочка $\sigma$ оказьвается композицией всего лишш двух фрагментов $\phi_{0}$ и $\phi_{1}$. В качестве пробной поверхности цепочки $\sigma$ мы можем принять поверхность $S=S_{1} \cup p\left(S_{0} \backslash \omega_{m}\right)$. Эта поверхность является пробной, поскольку ее индекс пересечения с $\gamma_{n}$ равен -1 и она имеет нулевой индекс пересечения с каждой из сфер $\omega_{0}, \ldots, \omega_{m-1}, \gamma_{0}, \ldots, \gamma_{n-1}$. Поясним это. Никакая из сфер $\omega_{0}, \ldots, \omega_{m-1}$ в силу леммы 1 не может пересекать никакую из сфер $\left\{\gamma_{i}\right\}$, в частности, и сферу $\gamma_{0}$. И потому $S$ имеет нулевое пересечение со сферами $\gamma_{0}, \ldots, \gamma_{n-1}$. Далее, в силу леммы $3 y_{0}=p$, т.е. сфрера $\gamma_{0}$ входит в $S_{1}$ с множителем $p$. Поверхность $S_{0} \backslash \omega_{m}$ включена в $S$ также с коэффициентом $p$. Поэтому при $i<m$ сфера $\omega_{i}$ имеет с поверхностью $S$ такой же индекс пересечения, как и $p$ раз взятая поверхность $S_{0} \backslash \omega_{m}$, и потому из определения пробной поверхности следует, что это равно нулю.

По условию леммы $\omega_{m}^{2}=-1$, и, следовательно, для фрагмента $\phi_{1}$ соответствующий определитель $d=1$, и потому в силу леммы $3 S_{1}^{2}=-p q$ и $H=p+q-1$. Опять же, из условия $\omega_{m}^{2}=-1$ получаем $\left(p \omega_{0}\right)^{2}=-p^{2}$ и, следовательно,

$$
S^{2}=p^{2}\left(S_{0} \backslash \omega_{m}\right)^{2}+S_{1}^{2}=S_{0}^{2} p^{2}+p^{2}-p q .
$$

Из тех же соображений получается, что $H=H_{0} p-p+H_{1}=H_{0} p+q-1$. 
Пусть теперь $l \neq 0, S_{2}$ - пробная поверхность композиции фрагментов $\phi_{0}$ и $\phi_{1}$, и $H_{2}-$ значение класса Черна на $S_{2}$. Полученные выше равенства для $S^{2}$ и $H$ мы можем теперь переписать так: $S_{2}^{2}=S_{0} p^{2}+p^{2}-p q$ и $H_{2}=H_{0} p+q-1$.

Запишем пробную поверхность композиции всех трех фрагментов в виде

$$
S=\bigcup_{i=0}^{m+n+l} n_{i} \omega_{i}
$$

В силу леммы $2, n_{i}=1$ при всех $i \geqslant m+n$. Заметим, что поверхность $\bigcup_{i=0}^{m+n} n_{i} \omega_{i}$ можно рассматривать как пробную поверхность композиции первьх двух фрагментов. Из формул, приведенньх в утверждении 2, видно, что включение в композицию третьего фрагмента уменьшает на $l$ значение $S^{2}$ и увеличивает на $l$ значение $H$. Это и приведенные вьше формулы дают искомые равенства. Лемма доказана.

ЛЕмма 7. Числа $H, S^{2}, H_{0}, S_{0}^{2}$ (см. обозначения перед леммой 6) удовлетворяют соотношениям:

1) если $S^{2} \geqslant 1$, mo $S_{0}^{2} \geqslant 1$;

2) если $H_{0}+S_{0}^{2} \geqslant-2$ u $S^{2} \geqslant 1$, mo $H+S^{2} \geqslant-2$;

3) если $H_{0}+S_{0}^{2}>-2 u S^{2} \geqslant 1$, mo $H+S^{2}>-2$;

4) если $H_{0}+S_{0}^{2}=H+S^{2}=-2$, mo $H_{0}=-3 u S_{0}^{2}=1$.

ДоказАТЕльство. Так как $d=1$, то в силу леммы $3 q \geqslant p+k \geqslant p+1$. В силу леммы 6 (см. п. 1) имеем $S_{0}^{2}=p^{-2}\left(S^{2}-p^{2}+p q+l\right)$. Следовательно, $S_{0}^{2}>0$, а так как это число целое, то $S_{0}^{2} \geqslant 1$.

В силу леммы $6 H+S^{2}$ не зависит от $l$, поэтому далее, оценивая это выражение, мы сначала укоротим цепочку так, чтобы $l$ стало равньп 0 , получим нужную оценку, удлиним цепочку до прежнего размера и скажем, что полученная оценка сохраняется и в общем случае. Это позволительно, поскольку присоединение элементарной цепочки не изменяет значения $H+S^{2}$. Так как при $l=0$ число $S^{2}$ делится на $p$, его можно записать в виде $S^{2}=n p$. В пш. 2 и 3 предполагается, что $S^{2} \geqslant 1$ и потому $n \geqslant 1$. Складьвая равенства из леммы 6 , поделив предварительно первое из них на $p$, получаем $n+H=\left(S_{0}^{2}+H_{0}\right) p+p-1$. В формулировке п. 2 предполагается, что $H_{0}+S_{0}^{2} \geqslant-2$, и потому $n+H+p-1 \geqslant-2$. А так как $n p \geqslant n+p-1$ при $n \geqslant 1$, то $H+S^{2}=n p+H \geqslant-2$. Пункт 2 леммы доказан. Пункт 3 леммы доказьвается аналогично.

Докажем п. 4, считая теперь $l \geqslant 0$ произвольньм. Складьвая равенства из леммы 6 и учитьвая условие $H+S^{2}=-2$, мы видим, что число $q+1$ делится на $p$. Но в силу леммы $5 p+2 \leqslant q+1 \leqslant 2 p$, и потому из указанного условия делимости получаем, что $q=2 p-1$. Подставляя последнее в сумму равенств из леммы 6 и учитывая условие $H+S^{2}=-2$, получаем $S_{0}^{2} p^{2}+H_{0} p-p^{2}+3 p=0$. Так как $H_{0}+S_{0}^{2}=-2$, мы можем записать эти слагаемые в виде $S^{2}=1+\varepsilon$ и $H=-3-\varepsilon$. Получается $\varepsilon\left(p^{2}-p\right)=0$. Но $p \geqslant 2$, поскольку $k \geqslant 1$; следовательно, $\varepsilon=0$, и мы получаем $H=-3$, а $S^{2}=1$. Лемма доказана.

\section{6. Доказательство теоремы.}

Пусть $\sigma=\sigma_{0} \ldots \sigma_{l}(l \geqslant 1)$ - минимальная цепочка. Всякую такую цепочку можно представить в виде композиции фрагментов $\phi_{1} \ldots \phi_{n}$, обладающих следующими свойствами: фрагмент $\phi_{1}$ состоит не менее, чем из двух сфер, и он является или элементарной цепочкой или композицией элементарной цепочки и составной цепочки. При всяком 
$i>1 \phi$ рагмент $\phi_{i}$ является композицией составной цепочки и элементарной цепочки и при этом допускается, что эта элементарная цепочка состоит лишь из одной сферы. В этом случае $\phi_{i}$ состоит из одной составной цепочки.

Пусть $S_{i}$ - пробная поверхность композиции фрагментов $\phi_{1}, \ldots \phi_{i}$ и $H_{i}$ - значение первого класса Черна на $S_{i}$. Положим $S=S_{n}$ и $H=H_{n}$.

Пусть $H=-3$ а $S^{2}=1$. Покажем, что в этом случае каждьй из фрагментов $\phi_{i}$ является треугольной цепочкой и при этом $\widetilde{\sigma}_{0}^{2}=1$. Так как $S^{2}=1$, в силу леммы 7 $S_{i}^{2} \geqslant 1$ при всех значениях $i$.

Заметим теперь, что фрагмент $\phi_{1}$ при сделанных предположениях не может состоять только из элементарной цепочки, поскольку в этом случае оказалось бы, что $S_{1}^{2} \leqslant 0$. Получается, что фрагмент $\phi_{1}$ является композицией составной и элементарной цепочек. Пусть $p_{1}$ и $q_{1}-$ параметры этой составной цепочки, а длина элементарной цепочки равна $l_{1}$. Из леммы 3 следует, что $S_{1}^{2}=-d p_{1} q_{1}-l_{1}$ и $H_{1}=d\left(p_{1}+q_{1}\right)-1+l$. Так как $S_{1}^{2} \geqslant 1$ и $l_{1} \geqslant 0$, то $d=-\widetilde{\sigma}_{0}^{2}=-1$. Следовательно,

$$
H_{1}+S_{1}^{2}=p_{1} q_{1}-p_{1}-q_{1}-1=\left(p_{1}-1\right)\left(q_{1}-1\right)-2 \geqslant-2 .
$$

Поэтому в силу леммы 7 (п. 2) $H_{i}+S_{i}^{2} \geqslant-2$ при всех значениях $i$. Но $H_{n}+S_{n}^{2}=H+$ $S^{2}=-2$, и потому в силу леммы 7 (п. 3) $H_{i}+S_{i}^{2}=-2$ при всех значениях $i$. И, наконец, в силу леммы 7 (п. 4) получаем, что $H_{i}=-3$ и $S_{i}^{2}=1$ при всяком $i$.

Из двух последних равенств следует, что всякий из фрагментов $\phi$ является треугольной цепочкой. Действительно, обозначая через $p_{i}, q_{i}$ и $l_{i}$ параметры фрагмента $\phi_{i}$ и складывая выражения для $H_{i}$ и $S_{i}^{2}$ (см. лемму 6), получаем, что при всех значениях $i$, включая значение $i=1$, число $q_{i}+1$ делится на $p_{i}$. Но в силу леммы $5 p_{i}+2 \leqslant q_{i}+1 \leqslant 2 p_{i}$ и, следовательно, $q_{i}=2 p_{i}-1$. И опять же в силу леммы 5 получается, что составная цепочка фрагмента $\phi_{i}$ является составной цепочкой простейшего типа. Обозначим через $k_{i}$ порядок этой цепочки.

Мы можем вычислить теперь числа $l_{i}$. При $i=1$ имеем $S_{1}^{2}=p_{1} q_{1}-l_{1}$. Так как $d=-1$, то в силу леммы $5 q_{1}=1$ и $p_{1}=k_{1}+1$. Поэтому $l_{1}=p_{1} q_{1}-S_{1}^{2}=k_{1}$. Получилось, что фрагмент $\phi_{1}$ является треугольной цепочкой.

Далее, при $i>1$ в силу леммы $5 q_{i}=2 p_{i}-1$ и $p_{i}=k_{i}+1$. И потому, используя первое равенство леммы 6 , получаем $l_{i}=S_{i-1}^{2} p_{i}^{2}+p_{i}^{2}-p_{i} q_{i}-S_{i}^{2}=k_{i}$. Таким образом, при всяком $i$ фрагмент $\phi_{i}$ является треугольной цепочкой.

Вторая часть теоремы получается совсем просто. Если все фрагменты являются треугольньми цепочками и $d=-1$, то $q_{1}=1$ и при $i>1 q_{i}=2 p_{i}-1$ и при всех $i p_{i}=k_{i}+1$ и $l_{i}=k_{i}$. Поэтому индукцией по $i$ из леммы 6 получается, что при всяком $i S_{i}^{2}=1$ и $H_{i}=-3$. В частности, для пробной поверхности всей цепочки получается, что $S^{2}=1$ и $H=-3$. Теорема доказана.

7. Доказательство утверждения 1. Пусть для начала $\sigma$ - это элементарная цепочка. Тогда в ее представлении в виде $\sigma=\bigcup_{i=0}^{l} n_{i} \omega_{i}$ каждый из коэффициентов $n_{i}$ равен 1 (см. лемму 2). Поэтому из формул для $H$ и $S^{2}$, приведенных в утверждении 2 (см. раздел 3 ), следует, что $H+S^{2}=d-1$. Следовательно, в рассматриваемой ситуации $d=1$, т.е. $\widetilde{\sigma}_{0}^{2}=1$. При этом из указанной формулы для $H$ следует, что $l=H+3$. Таким образом, для случая элементарной цепочки утверждение доказано.

Рассмотрим еще один частньй случай. Пусть $\sigma$-это композиция одной составной цепочки порядка $k$ с параметрами $p, q$ и $d=1$ (т.е. $\widetilde{\sigma}_{0}^{2}=1$ ) и одной элементарной 
цепочки длины $l$. Так как для цепочки $\sigma$ дано $H+S^{2}=-2$, то и для рассматриваемой составной цепочки $H+S^{2}=-2$, и потому в силу леммы $3 p q-p-q-1=-2$. А так как $p \neq 1$, то $q=1$. Следовательно, в силу леммы $5 p=k+1$, и потому для всей цепочки $\sigma$ величина $H$ равна $H=-p-q-1+l$ и, следовательно, $l=k+H+3$. Таким образом, цепочку $\sigma$ можно рассматривать как композицию 3 -х фрагментов: составной цепочки простейшего типа порядка $k$, элементарной цепочки длины $k$ и еще одной элементарной цепочки длины $H+3$. То есть $\sigma$ является композицией одной треугольной цепочки и элементарной цепочки длины $H+3$.

Теперь, исключив два рассмотренных частных случая, мы можем считать, что цепочка $\sigma$ является композицией 3 -х фрагментов $\phi_{0}, \phi_{1}$ и $\phi_{2}$, которые удовлетворяют всем условиям леммы 6. (При этом допускается, что любой из фрагментов $\phi_{0}, \phi_{2}$ состоит из одной сферы с индексом самопересечения -1.) Складьвая равенства из леммы 6, разделив предварительно первое из них на $p$, и далее, разделив полученное на $p$, запишем результат в виде $H_{0}+S_{0}^{2}+1=\left(\left(S^{2}+l\right) / p+H-l+1\right) / p$. Кроме того, значение левой части - это целое нечетное число, поскольку из утверждения 2 (см. раздел 3 ) следует, что число $H_{0}+S_{0}^{2}$ четно.

Для упрощения записи положим $l-H-2=x$. Из сказанного следует, что числа $p$ и $x$ удовлетворяют следующим 4-м условиям:

1) $p \geqslant 2$

2) $x$ делится на $p$;

3) число $x / p-x-1$ делится на $p$;

4) число $(x / p-x-1) / p$ - нечетно.

Докажем теперь, что $l \geqslant H+3$. Для этого достаточно показать, что если число $x$ принимает одно из значений $-5, \ldots,-1,0,1$, то хотя бы одно из четырех указанных условий не будет вьполнено. Делается это непосредственной проверкой. Считаем, что первое условие вьполнено. При $x= \pm 1$ не вьполняется условие 2 . При $x=0,-3,-5$ не выполняется условие 3 . При $x=-2$ не выполняется условие 4 . При $x=-4$ возможны 2 варианта: $p$ равно или 2 или 4 . В обоих случаях не будет вьполняться условие 3 .

Так как $l \geqslant H+3$, то мы можем укоротить цепочку на $H+3$ элемента. Стянем в точку эти $H+3$ сферы. Особой точки у объемлющего пространства не появится, поскольку на каждом шаге стягивается сфера, индекс самопересечения которой равен -1 . Для полученной после стягивания цепочки $S^{2}=1$ и $H=-3$. Поэтому в силу теоремы из раздела 2 , она является композицией треугольных цепочек. Следовательно, цепочка $\sigma$ является композицией треугольных цепочек и одной элементарной цепочки длины $H+3$. Утверждение доказано.

\section{СПИСОК ЦИТИРОВАННОЙ ЛИТЕРАТУРЫ}

[1] Витушкин А. Г. Некоторые примеры в связи с проблемой обращения полиномиальных отображений $\mathbf{C}^{n} / /$ Изв. АН СССР. Сер. матем. 1971. Т. 35. С. 269-279.

[2] Оревков С. Ю. Диаграммы Рудольфа и аналитическая реализация накрытия Витушкина // Матем. заметки. 1996. Т. 60. № 2. С. 206-224.

[3] Витушкин А. Г. Описание гомологий разветвленной накрьвающей над $\mathbf{C}^{2} / /$ Матем. заметки. 1998. Т. 64. №62. С. 839-846.

Математический институт им. В. А. Стеклова РАН 